\title{
Monzer Fanun (Ed.): Colloids in Drug Delivery
}

\author{
Neil C. Henney
}

Published online: 21 September 2011

(c) Springer-Verlag 2011

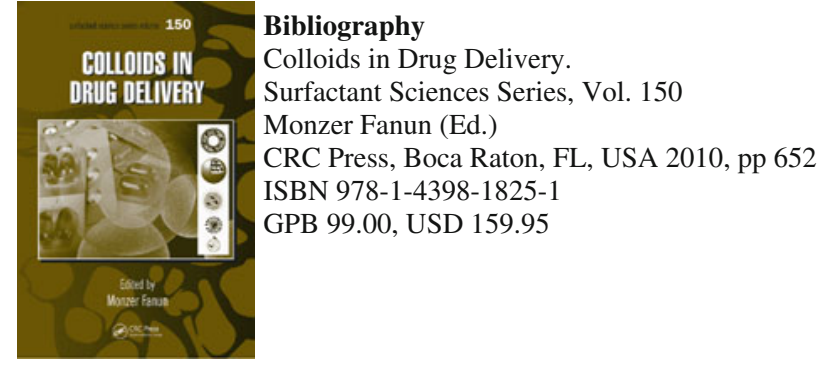

This expansive textbook is one of a number of recent works that delve into the field of colloids, surfactant molecules and attachable nanoparticles (such as in PEGylation), describing their physicochemical properties and their uses in a wide range of applications in mechanical engineering, cosmetics and in food textures and stability, to name but a few. Where this book differs is in its concentration on the considerations and applications of colloids in the science of pharmaceutics which itself extends to a sizeable volume. The business of manufacturing medicinal products that have a good 'shelflife', which allow reasonably simple dose measurements (of even distribution), which perhaps can be combined with other products that do not cause discomfort to the patient, which may target the delivery of the active ingredient to a specific organ, and so on, is no mean feat. Here the authors consider these problems in turn as we progress through chapters organised by the different uses and delivery methods offered by each type of colloidal technology.

The 25 chapters are written by contributors from an author list of 65 pharmaceutical scientists, all of whom are academics from universities across the world. Each chapter

N. C. Henney $(\bowtie)$

The School of Pharmacy and Biomolecular Sciences, Liverpool John Moores University, Liverpool, UK

e-mail: N.C.Henney@1jmu.ac.uk offers a description of the physicochemical properties of the technology, methods for the preparation, and of course considers the often multiple uses for the technology in designing drug delivery systems. The chapters vary in their emphasis on background science versus application, which may reflect the interests of the individual authors as much as the descriptive content itself, and similarly the organisational structure of each chapter differs. But this works well and is not a criticism. Diagrams and figures are used to good effect throughout the chapters, but are all in black-andwhite and sometimes few and far between. A number of the figures might be a little clearer in colour, but remain useful nonetheless and are of good resolution and size. On the whole, the chapters are text-heavy and full of numerical values, from infrared spectra to zeta potentials, particle diameters to $\log$ Ps. Again, this is an observation rather than a criticism. As an academic reference, the level of detail is welcomed. Each chapter includes extensive citations and a long reference list, where the reader can seek further detail.

A number of chapters end with the authors' observations and consideration of 'future directions'. As many of these technologies are relatively new (and some are cutting edge), speculation on their applications in drug delivery gives the reader an insight into how problems are being worked through to try to target diseases more effectively. Perhaps, nowhere is this more apparent than in developing effective and safe treatments for cancers, and of course this is discussed in a number of chapters and particularly in Chapter 24. The delivery of drugs via the pulmonary route also gets considerable attention in Chapters 21 and 22. The final chapter deals with the advancements the technologies offer to medical imaging, bringing possible improvements to diagnostics and surgical procedures.

This book is a well-written, detailed academic reference that would likely be of interest and value to industrial and academic pharmacists and pharmaceutical scientists. 